\title{
Collaborative Competencies in Professional Social Networking: Are students short changed by the curriculum in Business Education?
}

\author{
Benson, Vladlena ${ }^{a}$ and Filippaios, Fragkiskos ${ }^{b}$ \\ a. Kingston Business School, Kingston University London, Kingston upon Thames, KT2 7LB, email: \\ v.benson@kinsgton.ac.uk \\ b. Kent Business School, University of Kent, Canterbury, email: F.Filippaios@ kent.ac.uk
}

\begin{abstract}
Social networks have earned their indisputable place as a collaborative learning tool in education. In this paper, we address the gap in literature on developing a set of competencies to allow graduates to make the most of collaborative technologies for business and professional activity as well as career management. Based on a survey of over 600 business school graduates from AMBA accredited UK universities, we are able to identify the level of awareness and degree of application of professional usage of social networks amongst students. Our results show that work experience and age of the business students play a significant role in their usage of social networks for professional purposes, knowledge and career management. The data shows that younger students are more social networking savvy when it comes to identifying business opportunities, while older graduates are less confident. This is important as traditionally graduate students are individuals in their mid careers and the skill gap between generation $\mathrm{Y}$ and the experienced graduates need to be bridged by adequate curriculum changes.
\end{abstract}

Keywords: Collaborative learning environments; professional networking; career management; graduate competencies; curriculum design.

\section{Highlights:}

- We address a set of graduate competencies for professional networking, including collaborative competencies for business and career development

- Results of a survey of 600 business school graduates from universities are presented

- Skill gap between generation $\mathrm{Y}$ and experienced graduates must be covered by curriculum changes

- We construct social networking skills taxonomy and present implications for practice

\section{Introduction.}

The last decade has seen online social networking take the centre stage amongst innovative collaboration tools. Higher Education was no exception. Academic literature provides a rich account of successful academic applications of social technologies. Earlier research showed the potential of social networking throughout the entire student lifecycle from pre-application to university to life-long learning stages (Benson, Morgan, \& Tennakoon, 2011) and provides examples of using social networks as means for collaborative knowledge sharing. Knowledge 
management literature stresses the importance of flexibility and collaborative competencies in knowledge sharing for business success (Lytras \& Ordóñez de Pablos, 2008). A number of studies provide an ample account of social technologies integrations into learning and teaching (for review see e.g. Benson \& Morgan, 2014), the area which has been transformed through the new technology medium. This transformation corresponds to the emergence of collaborative learning which is highly enriched by the potential offered by social technologies (Lytras \& Ordóñez de Pablos, 2011).

Users tend to join social networks as they perceive value in belonging to a network and building connections through it. For example, (Yang \& Lin, 2014) suggest that the value of belonging to a network is threefold. According to the authors, joining Facebook offers its users social, hedonistic and epistemic values, and users with different aims of belonging to a network draw different benefits from it. Furthermore, knowledge sharing opportunities through social networks have been explored by Chow \& Chan (2008). It was found that employees receive satisfaction from content and knowledge sharing in trusted environment in professional settings. This is closely linked with the concept of social capital built through the online social networks. Social capital theory supports the notion of ties between network entities bringing value to those connected. This is even more true in the current unstable economic climate with employment fluctuations and highly competetive job market for graduates (Gu, Zghang \& Liu, 2014).

The increased popularity of social networking is largely due to the proliferation of smart/mobile devices and the intuitive nature of social technologies (Croitoru et al., 2013; Salehman \& Negahban, 2013). An area which, until now, received limited coverage in the academic literature refers to the move from the intuitive use to knowledge led ability to leverage social networking sites for business and professional purposes. Earlier publications addressed the intuitive use of social networks and social capital accumulated through networking by graduates (Benson, Morgan, \& Filippaios, 2010). The researchers showed that international students were actively using networking with other social users to form useful connections during job search and career management, while UK students, especially undergradutes, used social networks largerly for personal and leisure use. Other publications call for more research on the professional competencies necessary to operate in social networking environment (Shah, 2010).

The accounts of universities providing comprehensive courses addressing skills for professional use of online social networks are scarce, even more so in the context of business and management programmes. Exploring the factors that influence business students' use of social networks for personal and business purposes is the key research question of this paper. Using a sample of more than 600 graduates from AMBA accredited schools we explore the role of demographic factors, years of work experience, digital footprint awareness and concerns over privacy and addiction on the use of social networks. More precisely, this paper provides an insight into the reasons why business students join social networks and what value they see in signing up to yet another social application.

The paper is structured as follows. It opens up with the review of literature on the successful application of social technologies, including knowledge sharing, and their impact which may hinder adoption of social media in academia. We then describe the method for empirical data collection and analysis in section 4 . Section 5 outlines the findings on the awareness of professional use of social networks, which is followed by implications for practice. The paper concludes with possible limitations of the study and open research questions. 


\section{Implications of social media in personal and professional settings for graduates}

Reports of social network adoption in learning and teaching are plentiful (Pimmer, Linxen, \& Grohien, 2012) and knowledge management literature emphasises the need for development of collaborative competencies in graduates, especially in the business education area. The use of social networks as collaborative platforms in education has far reaching implications. As discussed in Benson et al. (2011), social networks represent a knowledge convergence platform for students at different stages of Higher Education and have the potential to enable collaboration and integration into life-long learning. Development of collaborative competencies, knowledge construction and sharing skills are important for successful graduate employment. Recent studies on human resource competencies show that organisational requirements for the exploitation of human resource capital require collative competencies for knowledge dissemination and sharing in the work place to promote organisational performance (Lytras \& Ordóñez de Pablos, 2008).

Along the same line of argument, studies have shown that the creation and strength of social ties can lead to knowledge contribution behaviour (Chai \& Kim, 2012) and social networks have now become the main means for communicating and keeping in touch with others to share and create knowledge (Johnston, Chen \& Hauman, 2013). It has also recently been shown that this knowledge creation and sharing process is enhanced by the amount of use of social networks, with people that make extensive use of social networks to show a higher willingness to share information (Chang \& Hsiao, 2014).

The main aim of this paper is to explore the factors that influence the use of social networks from graduates in personal and professional settings. We would expect certain demographic characteristics to significantly influence use, with age and gender being two factors explored in this paper. The literature is quite scarce with regard to the influence gender has on the use of social networks. Age acts as a good indicator of information technology literacy and usually younger people are more inclined to use social networks for personal and professional purposes (Haight, Quan-Haase \& Corbett, 2014). The study also has shown that generally women tend to join social networks in order to participate in collaborative knowledge creation. With the above arguments in mind we put forward our two first hypotheses:

H1: Age will have a negative effect on the use of social networks for personal and professional purposes.

H2: Women will have a stronger tendency to use social networks for personal and professional purposes.

The second major factor determining use of social networks for personal and primarily for professional purposes is the years of work experience of users. Years of work experience tend to suggest a maturity in terms of career development and an awareness of the factors that lead to success in the business place. Along the same line of argument, people with established careers will rely less on social networks to find a job. Contrary to this, people with established careers and less use of social networks might be less prepared for an emerging virtual professional culture (Agarwal \& Mital, 2009). Understanding this virtual professional culture might be crucial for the first few years of developing your career path. This leads to the development of our third hypothesis:

H3: Years of work experience will have a negative effect on the use of social networks for personal and professional purposes. 
Following on from years of work experience, time availability is another crucial factor that influence the use of social networks for personal and professional purposes. Usually people in senior positions in organisations with demanding jobs and possible issues of overexposure of information will tend to use social networks less. Studies (Weng, et al, 2012) have shown that the issues of overexposure pose a strong constrain to the use. This leads to the development of our fourth hypothesis:

H4: Time availability (lack of) will have a negative effect on the use of SNS for personal and professional purposes.

Clearly, the positive impact of Information and Communication Technology, including social media, outweighs its negative aspects. This explains why the literature on adverse effects of technology is relatively lean. According to recent studies, people feel pressured into checking their messages even when off work; ubiquitous technology creates a disruptive effect on both social life and productivity (Christakis \& Fowler, 2013). Research shows that social networks, Skype and instant messaging lead to stress induced by multitasking at work, which in turn leads to monetary losses for organisations (Baumer, 2013).

Factors such as perceived risk of social networks and the overall internet risk perception will negatively influence both the use as well as the enjoyment users draw from engagement (Chen, 2013). General concerns over privacy abuse will also contribute to users' perceived risk and therefore will negatively influence use. Finally, factors such as the social networks' structural assurance captured by regulations and code of conduct lead to knowledge contribution behaviour (Chai \& Kim, 2012).

Addiction has been linked to overuse of social media and is a cause of many other psychological implications including loss of confidence, decline of face to face communication skills and break up of personal relationships (Salehan \& Negahban, 2013). Users easily develop trust into social networks and their providers, this makes users predisposed to information disclosure behaviour to legitimate entities or those with malicious intents (Elmi et al., 2012). In earlier research trust has been long associated with propensity towards information disclosure online, this behaviour extends to social media. With the proliferation of social technologies into every area of human activity, this user friendly and high quality technology, as perceived by its users, leads to the development of higher levels of trust (Rose, 2011). Self-selection of the network membership, trust of the fellow network members promotes higher levels of personal information disclosure and threatens privacy. The above arguments lead to the development of our fifth and sixth hypotheses:

H6: Existence of privacy worries will negatively influence the use of SNS for personal and professional purposes

H7: Worries of addiction will negatively influence the use of SNS for personal and professional purposes

A final area that we wish to explore, especially on the use of social networks, for professional purposes primarily, is the awareness that one's digital footprint can have on career prospects. A number of studies suggest that employers make use of the information on social networks to make decisions on potential applicants and these do provide a good basis for future recruitment of employees (Clark \& Roberts, 2010; McLaughlin 2009). Despite the fact that it is still debatable as to whether students use social networks for job purposes (Herbold \& Douma, 2013), evidence clearly shows that companies that use campus based recruitment practices often use them to explore social behaviour of applicants such as drinking, use of 
recreational drugs or sexually explicit behaviour (Roberts \& Roach, 2009). This leads to the formulation of our last hypothesis:

H8: Awareness of the digital footprint will negatively influence the use of SNS for personal and professional purposes

It is evident that social networks facilitate collaborative networking and competency in their use and will become part of employability skills for the future. (Fernandez, 2012). Users of social networks need to be better informed about the privacy protection mechanisms and how service providers use their personal information, as they are more likely to reveal private information in this trusted setting. This applies to business graduates and professionals and needs to be addressed by relevant curriculum.

\section{Taxonomy of Social Networking Competencies}

Understanding implications of online behaviour and the significance of digital footprint is important for successful career management, professional networking and developing business opportunities online. Earlier research (Pempek et al., 2008) shows that students use SNS for one-to- many content dissemination ( broadcasting content to other users) and for building their identity. Students use traditional markers, including religion, political ideology, and media content to express their identity. Very few, however, have a clear understanding how their social media identity builds or damages student personal brand. Students are often left in the dark about online reputation, its attributes, consistency and professional extensions of social media activities. To develop strong social presence and reputation, students need to be aware of the potential of the participation in the networking events, interactions with peers, $\mathrm{CV}$, application forms, media content accumulation. This social media content is collected on personal accounts on professional SNS, such as LinkedIN, through university and its social media activity, workplace, even friends sharing their media elsewhere.

The online identity screening is imperative for graduates looking for a job or engaging in business relations online, but are students the only stakeholders in their reputation scrutiny on social media? The statistics of social media screening by prospective employers is brutal. According to a study of employers social media scrutiny practice by (Reppler.com, 2013) 69 $\%$ of organisations stated that they rejected candidates because of their social networking contents. Social networks used for screening are include Facebook, checked by $76 \%$ employers, twitter 53\% and LinkedIn 48\% respectively. According to the same source social media scrutiny takes place as soon as the job application is received by $48 \%$ employers, with smaller rates at the later stages of the job application process.

Building own online brand for graduates may include setting up a LinkedIN profile showing skills, expertise and media to external visitors, but not only that. Other SNS, such as YouTube or Instagram have opportunities for accumulating professional or creative content. Other strategies for developing professional social presence and extending personal brand (example of LinkedIN) include:

- Follow group discussions

- Become a thought leader, start ( and actively maintain) topical blogs

- Join companies for career planning research

- Regularly search for jobs www.linkedin.com/studentjobs. 


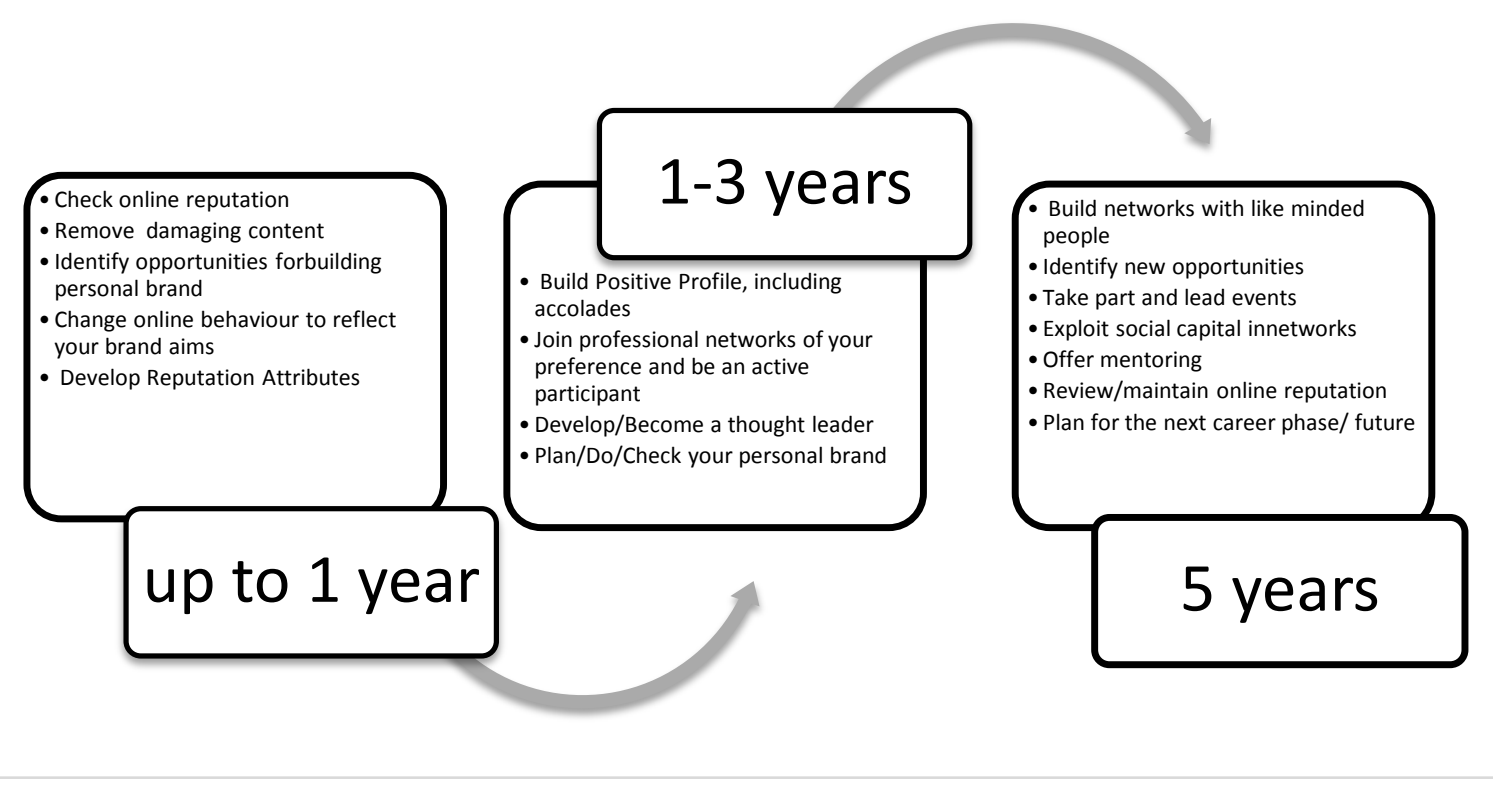

Figure 1. Long term evolvement of Graduate Social Media Competencies.

Those graduates with a positive impression of their personality and organisational fit, showcasing their achievements whilst at university, demonstrating creativity and strong communication skills through their social media profiles are more likely to be hired (Reppler.com, 2013). Setting up social media presence is only the start for graduates, social strategy for individuals need to be planned for and developed overtime as shown in figure 1 . Universities should play the leading role in building graduate competencies in the professional activity on social networks.

\section{Methodology and Sample Characteristics}

This study is a part of a wider research into the use of social networks by business students and graduates with data collected from questionnaires distributed between 2008 and 2012 in two AMBA accredited Universities in the UK and a direct questionnaire sent to AMBA members (primarily MBA students and graduates). The questionnaire was constructed following two focus groups and consists of a number of questions on participants' use of social networks and the internet. For the purposes of this study the whole sample was used as this covered a wide range of participants, from undergraduate students to executives studying towards an MBA. The overall sample distribution can be seen below in table 1 .

\section{Insert Table 1 here}

Our sample consists of 645 questionnaires with 354 undergraduate, 120 pre-experience masters and 171 MBA (post-experience Masters). The relevant averages for each group of the main variable used in our analysis, years of work experience can be seen in parentheses in Table 1. The overall average of work experience for our undergraduates is just over 3 years with the work experience being over 5 years for those pursuing a pre-experience Masters and over 11 years for those participants pursuing an MBA degree. Participants coming from the AMBA membership questionnaire show a slightly higher work experience with an average of 12,5 years which is almost 3 years higher than participants coming from the two AMBA accredited Schools. 
In table 2 we present the average age for each group and the proportion of female to male participants. As it can be seen the undergraduate students have an average age of just above 21 years whilst the average age is 27 and 33 for the pre-experience and the MBA participants respectively. The proportion of $60 \%$ female participants for the undergraduate studies drops to almost half $(34.50 \%)$ for the MBA group. This is not surprising and it is in line with the general trend in higher education. Again participants from the AMBA membership questionnaire are older by an average of 3 years but also show a stronger female participation rate close to $40 \%$.

\section{Insert Table 2 here}

This study follows a twofold quantitative approach. In our first step we explore differences in our participants' use of social networks, the reasons that drive their engagement and their use of Facebook versus LinkedIn. In all cases, we associate their behaviour with their work experience and we explore the statistical significance of the difference through the use of ANOVA technique.

In our second step, we explore further the reasons that influence their use of social networks for business purposes through the use of an ordered logistic regression. The use of an ordered logistic regression analysis was preferred over an ordered probit on the basis of the log likelihood function. Our dependent variable was constructed though the use of a Cronbach's alpha between the two instruments used to measure the business use of social networks. These two instruments corresponded to participants' agreement on using SNS for finding a job and finding business. The Cronbach's alpha coefficient was 0.91 which indicates an excellent internal consistency. Our independent variables corresponded to the years of work experience, the age and the sex of the participants. In our regression analysis, we have also used a variable measuring the factors that prevent participants for taking part in social networks all together which was created through a Cronbach's alpha of the factors presented in Table 5 and had a value of 0.79 which also indicates a high level of internal consistency. Finally, we have also used certain factors from Table 5 as independent variables to examine how much worries about the use of internet and worries about the potential digital footprint are preventing participants from making further use of social networks. The results of our logistic regression are presented in Table 6.

\section{Results and Discussion: Taxonomy of Social Networking Skills}

The current section presents the main results of our paper and offers the starting point for the creation of a taxonomy of social networking skills. As a first step, we enquired participants regarding their use of social networks for business and social purposes. Table 3 reports our findings according to participants average work experience (in years). We have also conducted an ANOVA analysis in each case and we report the corresponding F-statistic and its level of significance.

\section{Insert Table 3 here}

Firstly, it is interesting to note that the use of social networks for business purposes is increasing with the average years of work experience. Participants with less than 4 years of work experience are either using social networks less or not at all for business purposes in comparison to when they first joined. In contrast, participants with significant work experience (more than 6 years) are currently using social networks more for business purposes. The results of our ANOVA analysis confirm the significant difference between groups. On the contrary, there is no association of work experience and use for social 
purposes. Differences between different levels of groups are actually very small and not significant. This offers partial support to our H3 and clearly indicates that the years of work experience have a different effect on the personal and professional use of social networks.

Secondly, we explored participants' frequency in logging in social networks for business and social purposes. Although in both cases significant differences were found with regards to our AVONA analysis the actual behaviour is different for business and social use. We observe a clear trend of participants with high levels of work experience to be medium frequency users for business purposes, logging in primarily on a daily and weekly basis. This indicates a Ushaped curve between logging in frequency and work experience. Contrary to this, the relationship is linear when it comes to social use. The frequency of logging in is diminishing as years of work experience increase. Participants with very high levels of work experience (more than 8 years) are hardly ever or never log in to social networks for social use.

Thirdly, we wanted to explore the level of engagement with social networks for business and social use. We, therefore, asked participants how often they take part in discussions. Results for business use indicate that there is no clear association between the level of activity and years of work experience whilst there is again a clear negative and statistically significant association between the level of activity in discussions and years of work experience for social purposes. This indicates that more experienced participants tend to avoid taking part in discussions for social purposes and this perhaps reflects their key focus on the business use of social networks.

Finally, following on from the above we wanted to explore participants' frequency of updating their profile. There is a clear distinction between participants with low levels of work experience and high levels of work experience for business use with the former updating their profile quite frequently (daily and weekly) and the later less frequently (monthly or less often). This perhaps is related to the stage of career maturity of the latter and the reduced need to update their profile. Again, a clear linear trend emerges for social use with update frequency dropping as work experience rises.

From the above, it is evident that participants with work experience use social networks more for business purposes but they are also savvy of the impact of social networks on their time commitments as well as on their public profile which can be accessed by others, including potential future employers. These findings offer strong support to $\mathrm{H} 4$ and $\mathrm{H} 8$ confirming the effects of time availability and awareness of digital footprint on the use of social networks.

As a second step we wanted to focus more our attention on the two dominant social networks reported by participants, i.e. Facebook and LinkedIn and explore the reasons that drive participation in those two networks. Table 4 presents the results with regards to the key social and business reasons driving participation and the average work experience in years.

\section{Insert Table 4 here}

With regards to the first driver (To be generally sociable) and Facebook there is a clear association between participants' level of agreement with this statement and their years of work experience. Contrary, no such statistically significant association was found for LinkedIn. It is worth noting though that the average profile of participant for Facebook has almost half of the work experience of those participants of LinkedIn. These two findings reveal a clear understanding of participants' use of Facebook as a social network site whilst cast some doubt over participants understanding of the use and purpose of LinkedIn as a network for social networking. This is not surprising and it is in line with the way the two 
social networks position themselves with Facebook focusing more on social aspects and LinkedIn more on business/networking aspects.

Results for peer pressure (both old and new friends) as a driver to join the two social networks are similar in pattern and show an association between participants' level of agreement with this statement and their work experience. We can, thus, confidently claim that peer pressure is a strong driver for participation in both social networks. We also explored participants' perspective with regards to business oriented drivers, i.e. find a job and find business. With regards to the first there is a clear association with work experience. More experienced participants clearly understand the primary use of Facebook as a social network and thus disagree more with its use to find a job. It is interesting to note that only participants with less than 3 years of work experience (these correspond primarily to undergraduate students) show a strong agreement that Facebook can be used to find a job. A mixed picture emerges for LinkedIn with participants with lower levels of work experience to either strongly agree or disagree whilst more experience participants have less strong views on the use of the network. Especially participants with significant work experience showed a neutrality to this statement which indicates that their use of LinkedIn is based on other motives rather than finding a job. Results for finding business are similar to the ones above for LinkedIn but there is an interesting lack of association between this motive and years of work experience for Facebook. This shows the potential business opportunities that could emerge for a primarily social network like Facebook. Facebook is more and more frequently used as a medium for advertising and marketing and this is reflected in our findings.

As a complement to the above, and in order to explore the validity of our H6 and H7, we also explored the factors that prevented participants from participating further to social networks. These results are presented in Table 5.

\section{Insert Table 5 here}

Although we did not distinguish between various social networks, in this case, results show a very interesting pattern. A number of factors were presented to participants and it was requested to highlight their level of agreement with each one of the statements. Again, we present the findings with regards to the level of work experience. The interesting findings of Table 5 are not only related to those factors that where ANOVA analysis demonstrates a clear association but also to the lack of significant association in a number of factors. This lack of significant association shows the lack of awareness of the digital footprint and the way this might be used by future employers as well as the need to educate students with regards to the use of social networks, irrespective of their years of work experience.

More precisely, participants identified the lack of time as a key factor. This factor clearly shows a linear relationship with the years of work experience with more experienced participants showing a stronger agreement. Similar to this, the need for information demanded is a key prevention factor, especially for experienced participants. Factors such as security worries and access to a computer do not differ significantly between participants. The most interesting result of Table 5 is, though, the lack of association between work experience and worries on other peoples' perceptions on participants' profiles and the information that future employees might have access to. One would expect that as participants gain work experience would develop an understanding of the way their digital footprint develops and this would be a key factor determining their use of social networks. In this case, our results suggest that such a behaviour does not take place and this is a key finding of our study. There is clearly a skills gap that needs to be covered with regards to the 
creation of awareness around the use of various SNS and how the information contained in one's personal profile can be used by third parties.

Our final step in the analysis of participants' use of social networks corresponds to the factors that drive the business related use. We created a composite measure of the two factors reported in Table 4 related to business use, i.e. find a job and find business. The Cronbach's alpha is 0.91 which confirms the validity of using a combined variable. We have used information on the most preferred social network reported by participants but results do not change for the second most preferred network. In Table 6 we present three models on the use of social networks with different factors as explanatory variables for participants' behaviour. We report the coefficients, the odds ration as well as the percent change in odds.

\section{Insert Table 6 here}

In model one, basic participants' demographic characteristics were used to explain the probability of using their preferred network for business use. It is evident that work experience and age are significant but operate in opposite directions. Participants with higher work experience tend to use more social networks for business purposes. This again partially verifies our $\mathrm{H} 3$ and clearly indicates the different effect of work experience when it comes to business use. It is also younger participants that use social networks for business purposes. This clearly supports our H1 confirming that age is a key factor for the participation in social network activity. The gender of participants though is not statistically significant for their use. It appears that the profile of the typical user of social networks for business purposes is the young professionals whilst more mature professionals tend to use social networks less for business purposes. Looking at the percent changes in odds ratios the effect of work experience is one and a half time of the one for age in increasing the probability and thus we can infer that this is the most important factor in our model.

In model two, we included a composite factor of the participants' responses to the statements presented in Table 5. The Cronbach's alpha for internal validity is 0.79 which is acceptable. This factor captures the existence of general concerns, from participants' side, which prevent further engagement. The higher this factor, the less the concerns of participants. It is evident from model 2 that our demographic characteristics keep their original significance and signs but this factor is also significant indicating that facilitating use by reducing participants' concerns could lead to a significant engagement for business purposes. The percentage change of odds ratio is quite large with an $86.8 \%$ change overall. This clearly verifies our H6 and $\mathrm{H} 7$ which correspond to factors that prevent the use of social networks.

Next, in model three, we decided to investigate, further, specific factors that prevent participants from using social networks and their effect on business use, specifically. The inclusion of these factors slightly changes the influence of demographics by turning the effect of age to a non-significant one. Results show that participants are not influenced by the lack of time when it comes to business use. Engaging with social networks for business purposes is so important that participants do not worry about their time commitments. This indicates that our $\mathrm{H} 3$ is not supported when it comes to the business use of social networks. Three factors were found to play a significant role. Lack of privacy, worries about what employers may find and worries about a potential addiction to the use of social networks. Results provide support to our $\mathrm{H} 7$ and $\mathrm{H} 8$ but not H6. With regards to the lack of privacy (H6), results indicate that the less this was a concern for participants the less they would use the social networks for business purposes. This is not unexpected, as lack of privacy might matter primarily for the social use where sensitive information can be made available to others. The 
results around potential worries about what future employers might find are also expected. Participants with less worries about this particular factor tend to engage more with social networks on a business use. This result should be seen in the light of the findings from Table 5. According to our previous findings, most participants were unaware of the effects of their digital footprint and thus this result emphasises even further the need for education initiatives that will train students and executives towards developing an appreciation of the use of social networks.

Finally, our results clearly suggest that those participants that worry less about becoming addicted do use social networks more for business purposes. Again this is not a surprising result as we would expect participants in general to use more social networks if they did not worry about the effect of this use on their lifestyle.

\section{Implications for Policy and Practice}

Results of our study have important implications for policy and practice. Lack of awareness of the impact of the digital footprint accumulated through social networking is worrying. It is necessary to address the skill gap of social networking usage for professional purposes and entrepreneurship.

Privacy concerns and security breaches concern social networking users and even more so in the context of commercial use of social media. Equipping business graduates with adequate understanding of the impact of the individual digital footprint and the business potential of social networks is imperative for $\mathrm{HE}$ institutions. Universities must include social media skill set development into the curriculum in order to widen professional and entrepreneurial opportunities for students. This inclusion should not only focus on the undergraduate studies but should span across all levels of education, including post-experience Masters, like the MBA. Curriculum should focus on generating awareness of the digital footprint and then include tools and methods of managing information and personal profiles in line with career aspirations.

\section{Limitations}

The survey discussed in this paper was administered to MBA students in UK AMBA accredited universities. Every effort to assure data purity and validity of responses has been made. However, it is conceivable that for a different sample of respondents results may be different. We also tested a student sample in UK institutions. We welcome further investigation and testing of the results in varies geographic locations and levels of study.

\section{Conclusions}

Advances in technologies have opened up new opportunities for collaborative learning and networking. Over the last decade younger and older generations alike embraced social technologies with enthusiasm and the extension of their use in educational setting was simply a matter of time. Recent literature draws a rich account of social networking application in education. Collaborative opportunities opened up by social media are unprecedented and examples of their applications in HE settings present opportunities for effective learning, group work and project management, new communication medium rivalling email, peer feedback and assessment tools; the list is almost endless. On the other hand uncertainty of higher education institutions rises with respect to allowing third party social networking applications, such as Facebook and Twitter, to run on university owned networks and channel personal data through them. Lack of legislative constraints, poor understanding of personal 
data handling procedures and cyber security threats targeting social media prevent universities from fully leveraging academic capabilities of social networks. Nevertheless, the opportunities for business and professional uses of social networks are experiencing steady growth and predictions of the arrival of 'social commerce', the next generation of e-commerce conducted through social channels, are becoming reality.

With these considerations in mind, we addressed the views of graduates in UK AMBA accredited institutions on their awareness of business and professional opportunities which social networks opened. The survey of 645 UK graduates of various backgrounds and nationalities revealed some significant gaps in realising business and professional opportunities through social networking activity and entrepreneurship. One of the results which emerged from the data analysis using a logistic regression was that the levels of awareness of social networking usage are not gender dependent. Both male and female graduates actively use social networks and have similar levels of knowledge with regards to their business applications.

Our findings suggest that a skill gap exists between younger and more mature students, which has important implications for practice. While Rogers (1995) classification of technology users into early adopters and laggards certainly has its merit, the role of university in bridging this skill gap between technically savvy generation Y and older graduates should manifest itself in offering appropriate curriculum for personal and professional development in this direction. The findings of our survey show that graduates with higher years of industry experience are more aware and are more likely to take advantage of business opportunities on social networks. Therefore it is important for higher education institutions to offer curriculum which facilitates peer learning and bring the social networking competencies to a higher level amongst all HE graduates.

This paper proposes a taxonomy of social networking usage skills for graduates developed over time. We offer this taxonomy for further empirical validation in more settings besides business graduates and universities. Our results have significant implications for policy and practice. Firstly, there is an existing necessity to address the skills of social networking usage among professionals and entrepreneurs. Secondly, there is a pressing need for policy makers and industry to develop legislative frameworks safeguarding individuals and personal when using social networks, especially for professional and commercial purposes. Finally, the education sector must add social networking competencies to the skill set developed in students in Higher Education stages and widen opportunities for academic staff to integrate social technologies into learning and teaching.

\section{References}

Agarwal, S, \& Mital, M (2009). 'An exploratory study of Indian university students' use of social networking web sites: implications for the workplace', Business Communication Quarterly, 72(1), pp. 105-110.

Baumer, S. (2013). Social Media, Human Connectivity and Psychological Well-being. The SAGE Handbook of Digital Technology Research, 71.

Benson, V., \& Morgan, S. (2014). Cutting Edge Technologies and Social Media in Higher Education. Hersheys: IGI Global. 
Benson, V., Morgan, S., \& Filippaios, F. (2010). Online Social Networks: Changing the Face of Business Education and Career Planning. International Journal of eBusiness , 40 (1), pp. 20-33.

Benson, V., Morgan, S., \& Tennakoon, H. (2011). Applications of Social Media in Student Lifecycle. International Journal of Knowledge and Learning, pp. 54-67.

Chai, S, \& Kim, M 2012, 'A socio-technical approach to knowledge contribution behavior: An empirical investigation of social networking sites users', International Journal of Information Management, 32(2), pp. 118-126

Chang, T, \& Hsiao, W 2014, 'Time Spent on Social Networking Sites: Understanding User Behavior and Social Capital', Systems Research \& Behavioral Science, 31(1), pp. 102-114

Chen, R 2013, 'Member use of social networking sites — an empirical examination', Decision Support Systems, 54(3), pp. 1219-1227

Chow, W., \& Chan, L.S.(2008) Social network, social trust and shared goals in organizational knowledge sharing, Information \& Management, 45(7), pp.458-465.

Christakis, N. A., \& Fowler, J. H. (2013). Social contagion theory: examining dynamic social networks and human behavior. Statistics in medicine, 32(4), 556-577.

Clark, L, \& Roberts, S 2010, 'Employer's Use of Social Networking Sites: A Socially Irresponsible Practice', Journal of Business Ethics, 95(4), pp. 507-525.

Croitoru, A., Crooks, A., Radzikowski, J., \& Stefanidis, A. (2013). Geosocial gauge: a system prototype for knowledge discovery from social media. International Journal of Geographical Information Science, 27(12), pp. 2483-2508.

Gu, J.,Zhang,Y., \& Liu, H.(2014) Importance of social capital to student creativity within higher education in China, Thinking Skills and Creativity,12 pp.14-25.

Fernandez, MS 2012, 'Blended teamwork: the Facebook experience', Business Education \& Accreditation, 4(1), pp. 33-48

Haight, M, Quan-Haase, A, \& Corbett, BA 2014, 'Revisiting the digital divide in Canada: the impact of demographic factors on access to the internet, level of online activity, and social networking site usage', Information, Communication \& Society, 17(4), pp. 503-519

Herbold, J, \& Douma, B 2013, 'Students' Use of Social Media for Job Seeking', CPA Journal, 83(4), pp. 68-71

Johnston, K, Chen, M, \& Hauman, M 2013, 'Use, Perception and Attitude of University Students Towards Facebook and Twitter', Electronic Journal of Information Systems Evaluation, 16(3), pp. 200-210

Light, B., \& McGrath,K. (2010) Ethics and social networking sites: a disclosive analysis of Facebook, Information Technology \& People, 23(4), pp. 290 - 311. 
Lytras, M.D \& Ordóñez de Pablos, P. (2008) Competencies and Human Resource Management: Implications for Organizational Competitive Advantage, Journal of Knowledge Management, 12(6), pp. 48-55

Lytras, M.D. \& Ordóñez de Pablos, P. (2011) Software Technologies in Knowledge Society, Journal of Universal Computer Science, 17 (9), pp. 1219-1221

McLaughlin, S. (2009) Facing the Facebook Nation: Will Social Networking Sites Help or Hinder Good Business Communications?, Public Relations Strategist, 15(2), pp. 17-19

Roberts, SJ \& Roach, T. (2009) Social Networking Web Sites And Human Resource Personnel: Suggestions For Job Searches, Business Communication Quarterly, 72(1), pp. $110-114$

Salehan, M., \& Negahban, A. (2013) Social networking on smartphones: When mobile phones become addictive. Computers in Human Behavior, 29(6), pp.2632-2639.

Shah, R. (2010). Social Networking for Business: Choosing the right tools and resources to fit your needs. Wharton School Publishing.

Pempek, T. A., Yermolayeva, Y. A., \& Calvert, S. L. (2009). College students' social networking experiences on Facebook. Journal of Applied Developmental Psychology, 30(3), pp. 227-238.

Pimmer, C., Linxen, S., \& Grohien, U. (2012). Facebook as a learning tool? A case study on the appropriation of social network sites from mobile phones in developing countries. BJET, 43 (5),pp. 726-738.

Reppler.com (2013) Managing Your Online Image Across Social Networks. Available at: http://blog.reppler.com/2011/09/27/managing-your-online-image-across-social-networks/. Accessed on $10 / 10 / 14$

Rogers, E. (1995). Diffusion of Innovations. New York: Simon \& Schuster.

Valenzuela, S., Park, N., \& Kee, K. F. (2009). Is there social capital in a social network site?: Facebook use and college students' life satisfaction, trust, and. Journal of ComputerMediated Communication, 14, pp.875-901.

Weng Marc, L, Ding Hooi, T, Puspitasari, M, Prasetya, I, \& Gunadi, S 2012, 'Overexposure in Social Networking Sites', Journal of Research for Consumers, 22, pp. 25-40

Wilson, R. E., Gosling, S. D., \& Graham, L. T. (2012). A review of Facebook research in social sciences. Perspectives on Psychological Science, 7, 203-220.

Yang, H., Lin, C. (2014) Why do people stick to Facebook web site? A value theory-based view, Information Technology \& People, 27(1), pp.21 - 37. 
Table 1. Sample distribution (count of participants and average years of work experience, in parentheses)

\begin{tabular}{|c|c|c|c|c|c|c|}
\hline & \multirow[t]{2}{*}{ Undergraduate } & \multicolumn{2}{|l|}{ Postgraduate } & \multirow[t]{2}{*}{ Postgraduate Total } & \multirow[t]{2}{*}{ Grand Total } \\
\hline & & & Pre-experience Masters & MBA & & \\
\hline & 2008 & & & & & \\
\hline \multicolumn{2}{|c|}{ University A } & $132(2.87)$ & $58(5.68)$ & $41(9.73)$ & $99(7.42)$ & $231(4.92)$ \\
\hline & 2012 & & & & & \\
\hline \multicolumn{2}{|c|}{ University B } & & $34(5.25)$ & $15(9.37)$ & $49(7.01)$ & $506.88)$ \\
\hline \multicolumn{2}{|c|}{ University A } & $221(3.22)$ & $28(4.96)$ & & $28(4.96)$ & $249(3.42)$ \\
\hline & 2012 & & & & & \\
\hline \multirow[t]{2}{*}{ AMBA } & & & & $115(12.47)$ & $115(12.47)$ & $115(12.47)$ \\
\hline & $\begin{array}{l}\text { Grand } \\
\text { Total }\end{array}$ & 354 (3.09) & $120(5.40)$ & $171(11.53)$ & $291(9.24)$ & $645(5.89)$ \\
\hline
\end{tabular}


Table 2. Sample Characteristics (Average age and proportion of female participants in the sample)

\begin{tabular}{|c|c|c|c|c|c|c|}
\hline & & Undergraduate & Postgraduate & & Postgraduate Total & Grand Total \\
\hline & & & Pre-experience Masters & MBA & & \\
\hline University A & & $21.62 / 53.79 \%$ & $27.62 / 51.72 \%$ & $31.60 / 21.95 \%$ & $29.27 / 39.39 \%$ & $24.90 / 47.62 \%$ \\
\hline University B & & & $26.41 / 64.71 \%$ & $30.71 / 33.33 \%$ & $27.66 / 55.10 \%$ & $27.53 / 56.00 \%$ \\
\hline University A & & $21.66 / 64.25 \%$ & $26.60 / 46.43 \%$ & & $26.60 / 46.43 \%$ & $22.22 / 62.25 \%$ \\
\hline \multicolumn{7}{|c|}{2012} \\
\hline
\end{tabular}


Table 3. Comparison of Participants Business and Social Use (by average years of work experience)

\begin{tabular}{|l|r|l|l|r|r|}
\hline Business Use & Mean & F-stat & Use now & Mean & F-stat \\
\hline Use now & 6.64 & & More & 4.06 & \\
More & 4.07 & & The same & 4.09 & \\
The same & 3.86 & & Less & 4.13 & \\
Less & 3.62 & & Not at all & 5.75 & \\
Not at all & 4.14 & $5.80 * * *$ & Total & 4.17 & 0.69 \\
Total & Mean & F-stat & Logging in & Mean & F-stat \\
\hline Logging in & 4.27 & & 2-3 times a day & 4.29 & \\
\hline 2-3 times a day & 6.56 & & Daily & 5.14 & \\
Daily & 7.84 & & Weekly & 5.97 & \\
Weekly & 5.88 & & Monthly & 5.90 & \\
Monthly & 4.81 & & Hardly Ever & 8.57 & \\
Hardly Ever & 5.19 & & Never & 17.42 & \\
Never & 5.98 & $3.51^{* * *}$ & Total & 5.53 & $14.90^{* * *}$ \\
Total & Mean & F-stat & Active part in discussions & Mean & F-stat \\
\hline Active part in discussions & 5.03 & & Always & 3.99 & \\
\hline Always & 5.43 & & Sometimes & 4.94 & \\
Sometimes & 6.99 & & Rarely & 5.73 & \\
Rarely & 5.78 & & Never & 7.48 & \\
Never & 6.03 & 1.22 & Total & 5.56 & $5.39 * * *$ \\
Total & Mean & F-stat & Update my profile & Mean & F-stat \\
\hline Update my profile & 3.38 & & Daily & 3.27 & \\
\hline Daily & 3.90 & & Weekly & 4.02 & \\
Weekly & 7.76 & & Monthly & 4.88 & \\
Monthly & 6.48 & & Less Often & 6.18 & \\
Less Often & 6.29 & $3.94 * * *$ & Total & 5.01 & $4.15^{* * *}$ \\
Total & & & \\
\hline
\end{tabular}

*** Statistically significant at $1 \%, * *$ Statistically significant at $5 \%, *$ Statistically significant at $10 \%$ 
Table 4. Participants' reasons for joining Facebook and LinkedIn (by average years of work experience)

\begin{tabular}{|c|c|c|c|c|}
\hline & \multicolumn{2}{|c|}{ Facebook } & \multicolumn{2}{|c|}{ LinkedIn } \\
\hline To be generally sociable & Mean & F-stat & Mean & F-stat \\
\hline Strongly Agree & 4.72 & & 6.79 & \\
\hline Agree & 5.53 & & 9.58 & \\
\hline Neither Agree or Disagree & 4.86 & & 11.43 & \\
\hline Disagree & 8.37 & & 11.38 & \\
\hline Strongly Disagree & 4.53 & & 8.32 & \\
\hline Total & 5.35 & $3.39 * * *$ & 9.98 & 1.77 \\
\hline Peer pressure (Old Friends) & Mean & F-stat & Mean & F-stat \\
\hline Strongly Agree & 3.52 & & 7.71 & \\
\hline Agree & 5.56 & & 8.93 & \\
\hline Neither Agree or Disagree & 5.18 & & 7.13 & \\
\hline Disagree & 6.55 & & 12.10 & \\
\hline Strongly Disagree & 4.57 & & 8.54 & \\
\hline Total & 5.13 & $3.77 * * *$ & 9.40 & $2.77 * *$ \\
\hline Peer pressure (New Friends) & Mean & F-stat & Mean & F-stat \\
\hline Strongly Agree & 3.05 & & 6.00 & \\
\hline Agree & 5.12 & & 8.60 & \\
\hline Neither Agree or Disagree & 5.64 & & 7.86 & \\
\hline Disagree & 6.76 & & 12.36 & \\
\hline Strongly Disagree & 4.50 & & 8.88 & \\
\hline Total & 5.17 & $4.89 * * *$ & 9.38 & $3.13 * *$ \\
\hline Find a job & Mean & F-stat & Mean & F-stat \\
\hline Strongly Agree & 2.75 & & 6.44 & \\
\hline Agree & 4.06 & & 10.53 & \\
\hline Neither Agree or Disagree & 4.07 & & 14.65 & \\
\hline Disagree & 6.03 & & 13.33 & \\
\hline Strongly Disagree & 5.15 & & 9.39 & \\
\hline Total & 5.06 & $3.00 * *$ & 9.52 & $6.42 * * *$ \\
\hline Find Business & Mean & F-stat & Mean & F-stat \\
\hline Strongly Agree & 3.42 & & 6.37 & \\
\hline Agree & 6.03 & & 10.79 & \\
\hline Neither Agree or Disagree & 4.05 & & 11.33 & \\
\hline Disagree & 5.50 & & 11.67 & \\
\hline Strongly Disagree & 5.17 & & 8.03 & \\
\hline Total & 5.03 & 1.60 & 9.40 & $3.44 * *$ \\
\hline
\end{tabular}

$* * *$ Statistically significant at $1 \%, * *$ Statistically significant at $5 \%, *$ Statistically significant at $10 \%$ 
Table 5. Reasons that prevent further engagement with SNS (by average work experience)

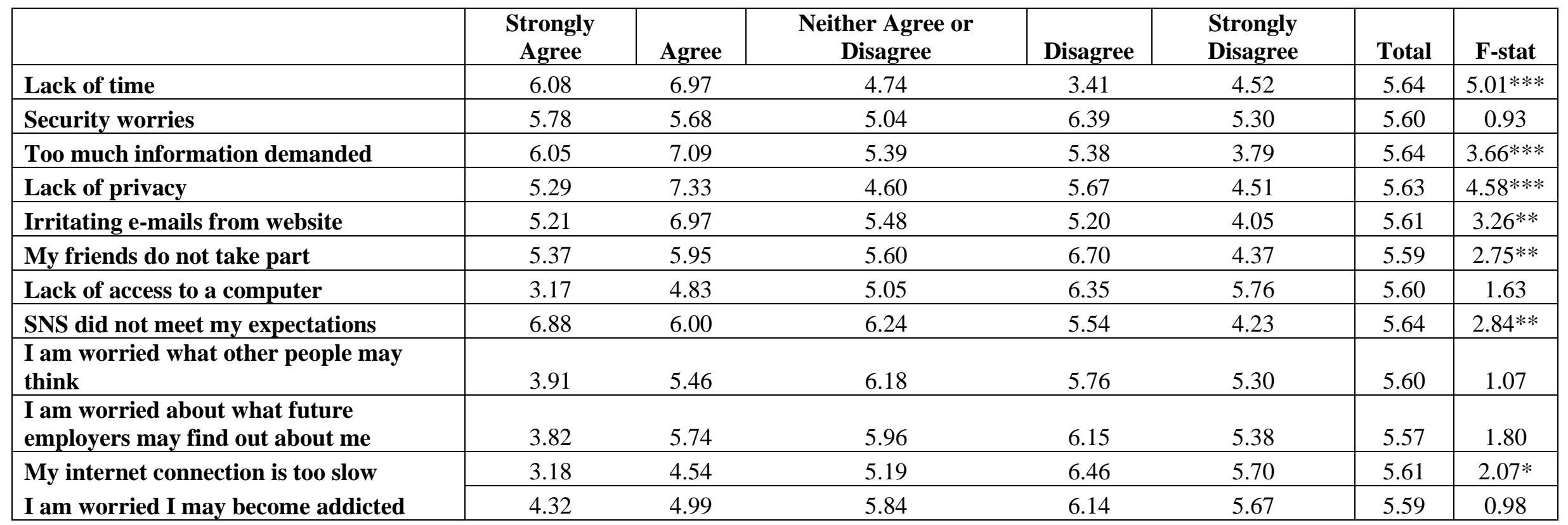

*** Statistically significant at $1 \%, * *$ Statistically significant at $5 \%, *$ Statistically significant at $10 \%$ 
Table 6. Ordered logistic regression (dependent variable: composite variable from Table 5 - Find a job \& Find business)

\begin{tabular}{|c|c|c|c|c|c|c|c|c|c|}
\hline & \multicolumn{3}{|c|}{ Model one } & \multicolumn{3}{|c|}{ Model two } & \multicolumn{3}{|c|}{ Model three } \\
\hline & Coefficient & $\begin{array}{l}\text { Odds } \\
\text { Ratio } \\
\end{array}$ & $\%$ & Coefficient & $\begin{array}{l}\text { Odds } \\
\text { Ratio }\end{array}$ & $\%$ & Coefficient & $\begin{array}{l}\text { Odds } \\
\text { Ratio } \\
\end{array}$ & $\%$ \\
\hline Work Experience & $\begin{array}{c}0.0600^{* *} \\
(0.0304)\end{array}$ & 1.062 & 6.2 & $\begin{array}{c}0.0623^{* *} \\
(0.0310)\end{array}$ & 1.064 & 6.4 & $\begin{array}{l}0.0548^{*} \\
(0.0325)\end{array}$ & 1.056 & 5.6 \\
\hline Gender & $\begin{array}{l}0.2916 \\
(0.2635)\end{array}$ & 1.339 & 33.9 & $\begin{array}{l}0.2047 \\
(0.1771)\end{array}$ & 1.227 & 22.7 & $\begin{array}{l}0.1131 \\
(0.1852)\end{array}$ & 1.12 & 12 \\
\hline Age & $\begin{array}{c}-0.0493 * \\
(0.0266)\end{array}$ & 0.952 & -4.8 & $\begin{array}{c}-0.0485^{*} \\
(0.0275)\end{array}$ & 0.953 & -4.7 & $\begin{array}{l}-0.0456 \\
(0.0281)\end{array}$ & 0.955 & -4.5 \\
\hline Prevention Factors & & & & $\begin{array}{c}0.6251 * * * \\
(0.1251)\end{array}$ & 1.868 & 86.8 & & & \\
\hline Lack of time & & & & & & & $\begin{array}{l}-0.0909 \\
(0.0796)\end{array}$ & 0.913 & -8.7 \\
\hline Lack of privacy & & & & & & & $\begin{array}{c}-0.1385^{*} \\
(0.0794)\end{array}$ & 0.871 & -12.9 \\
\hline Worry of employers & & & & & & & $\begin{array}{c}0.1902 * * \\
(0.0762)\end{array}$ & 1.21 & 21 \\
\hline Worry of addiction & & & & & & & $\begin{array}{c}0.3005^{* * *} * \\
(0.0753)\end{array}$ & 1.35 & 35 \\
\hline Log Likelihood & & -748.37 & & & 719.13 & & & -684.88 & \\
\hline Wald Chi2 & & 7.04* & & & $1.83 * * *$ & & & $3.19 * * *$ & \\
\hline Pseudo R2 & & 0.0204 & & & 0.0216 & & & 0.0237 & \\
\hline
\end{tabular}

Standards errors in parenthesis, *** Statistically significant at $1 \%, * *$ Statistically significant at $5 \%, *$ Statistically significant at $10 \%$ 